\title{
Use of Informatics in Identification of Adverse Drug Reactions
}

\section{Sachin Devi*}

Director, Laboratory of Experimental \& Clinical Toxicology, LECOM-School of Pharmacy, Bradenton, FL, 34203, USA

\section{What are Adverse Drug Reactions?}

Adverse drug reactions (ADRs) can be defined as, "Any response to a drug that is noxious and unintended, and that occurs at doses used in humans for prophylaxis, diagnosis, or therapy, excluding failure to accomplish the intended purpose." [1].

ADRs are commonly divided into two subtypes: 1) Type A and 2) Type B.

Type A ADRs are usually manifestations of exaggerated response of the known effect of the drug (e.g. bleeding from warfarin treatment). They are common and majority of them are attributed to pharmacokinetic or pharmacodynamic interactions. Most of these ADRs are predictable and therefore, preventable. On the other hand, type B ADRs are less common, serious and unpredictable. Majority of these types of ADRs are mediated by immune system and also termed as "hypersensitivity reactions".

Meta-analysis of prospective studies estimated that $6.7 \%$ of hospitalized patients have serious ADRs [2]. If these estimates are correct then ADRs are leading cause of death. It should be noted that these estimate do not include ADRs that occur in ambulatory setting. Even though there is no method that accurately measures the estimates of ADRs, there is general consensus that ADRs pose greater risk to public health. It should be emphasized that most of these ADRs are preventable.

\section{Why there are so many Incidences of Adrs and What is being done about them?}

These high incidences of ADRs raise logical question:why do so many ADRs occur in spite of thorough preclinical and clinical studies? There are several possible reasons. Here are few: 1) The number of physician visits has gone up 2) thenumbers of prescriptions have gone up 3) several patients take multiple medications. It has been demonstrated that the rate of ADRs increases exponentially after a patient is on 4 or more medications [3].

The next logical question is why ADRs are not identified before drugs are marketed to mass population? During preclinical trials, safety pharmacologists and toxicologists conduct several studies in order to identify potential ADRs including drug-drug interactions [4]. However, many of these studies are focused on major organ system and have limited impact to predict ADRs due to genetic or demographic variations. Additionally, there are millions of possible combinations of drugs given to the patients. Studying all of the drug-drug interactions during preclinical trials is not practical. Clinical trials are usually conducted on few hundred patients [5]. Therefore, rare ADRs (for example, 1 in 100,000 patient exposures) cannot be identified during clinical trials. These ADRs are generally identified only after millions of patients are exposed after marketing of these drugs to mass population. This underscores the importance and need for post-marketing surveillance.

Several measures have been implemented to identify and prevent ADRs after the drug has reached to the market. First and foremost is "Medwatch" program created by Food \& Drug Administration
(FDA). This is online ADR reporting system that encourages healthcare professionals to report suspicious serious ADR regardless of the causality. Additionally, many health systems have implemented electronic health records. There are several software programs available that assist healthcare professionals to prescribe and store patients' medical records. New generation computer software programs are able to detect and alert healthcare professional when combination of drugs with potential to cause ADRs are prescribed. It has been shows that optimal drug dosage with the help of computerized advice has beneficial effects on patient outcomescompared with empiric dosing without computer assistance) [6]. For example, managing therapeutics concentration of the drugs with narrow therapeutics window is challenging task. There are many software systems that have been designed to assist physicians to determine optimum drug dosage.

\section{Role of Informatics in Identifying and Preventing ADRs}

Large scale data collection hasgenerated vast amount of information. For example, healthcare professionals are encouraged to report possible ADR even if it is based on suspicion. This has created lot of noise and sifting through these datasets for the detection of possible ADR signal remains a challenge. Informatics thrives on these kinds of large datasets. Logical assumptions and hypotheses can assist in writing algorithms to develop software programs that can fade background noise and detect potential ADR signals.

Most of the software programs that are used as a part of electronic medical records systems are designed to generate alert in order to prevent errors. However, several studies indicated that frequent drug alerts result in "alert-fatigue" and can lead to overall ignorance of such alerts by healthcare professionals. Further research is needed to grade ADRs based on risk potential and to develop informatics programs that can generate only "high-value alerts". Additionally, several studies suggested that even though information is available, it is not optimally incorporated in decision-making process. Therefore, highly sophisticated informatics programs with better user interface must be designed to assist healthcare professional to sift through the plethora of information and utilize it in routine decision making process.

\section{References}

1. Karch FE, Lasagna $L$ (1975) Adverse drug reactions. A critical review. See comment in PubMed Commons below JAMA 234: 1236-1241.

2. Lazarou J, Pomeranz BH, Corey PN (1998) Incidence of adverse drug reactions in hospitalized patients: a meta-analysis of prospective studies. See comment in PubMed Commons below JAMA 279: 1200-1205.

*Corresponding author: Sachin Devi, Director, Laboratory of Experimental \& Clinical Toxicology LECOM-School of Pharmacy, Bradenton, FL, 34203, USA, Tel: 941-782-5941; E-mail: sdevi@lecom.edu

Received July 18, 2014; Accepted July 21, 2014; Published July 28, 2014

Citation: Devi S (2014) Use of Informatics in Identification of Adverse Drug Reactions. J Bioequiv Availab 6: e54. doi:10.4172/jbb.10000e54

Copyright: @ 2014 Devi S. This is an open-access article distributed under the terms of the Creative Commons Attribution License, which permits unrestricted use, distribution, and reproduction in any medium, provided the original author and source are credited. 
3. Jacubeit T, Drisch D, Weber E (1990) Risk factors as reflected by an intensive drug monitoring system. See comment in PubMed Commons below Agents Actions Suppl 29: 117-125

4. Hamdam J, Sethu S, Smith T, Alfirevic A, Alhaidari M, et al. (2013) Safety pharmacology--current and emerging concepts. See comment in PubMed Commons below Toxicol Appl Pharmacol 273: 229-241.
5. http://www.fda.gov/drugs/developmentapprovalprocess/ developmentresources/druginteractionslabeling/ucm110632.htm

6. Gillaizeau F, Chan E, Trinquart L, Colombet I, Walton RT, et al. (2013) Computerized advice on drug dosage to improve prescribing practice. See comment in PubMed Commons below Cochrane Database Syst Rev 11: CD002894. 